\title{
Ergonomic and Safety Experiences of Outdoor Gym Equipment Users
}

\author{
Merve Uca (Corresponding author) \\ Department of Physical Education and Sports Teaching, Faculty of Sport Sciences \\ Istanbul Aydin University, 34295, Istanbul, Turkey
}

Tel: 444-1-428-27502Ｅ-mail: merveuca@aydin.edu.tr

\begin{abstract}
Selim Yıldiz
Department of Physical Education and Sports Teaching, Faculty of Sport Sciences

Kocaeli University, 41001, Kocaeli, Turkey
\end{abstract}

Tel: 90-262-303-3630-3630Ｅ-mail: syildiz@kocaeli.edu.tr

Received: November 5, $2021 \quad$ Accepted: November 29, 2021

Published: December 31, 2021

doi:10.5296/jei.v7i3.19271 URL: https://doi.org/10.5296/jei.v7i3.19271

\begin{abstract}
The number of outdoor gym equipment (OGE) installed in parks has increased to encourage physical activity and social interactions among city dwellers. However, little is known about perceptions of and experiences with OGE ergonomics and safety. Therefore, this study aims to reveal people's ergonomic and safety experiences and perceptions about OGE. While the research universe consisted of individuals doing physical exercises in parks with OGE in Sakarya city, the sample was composed of a total of 359 people, 215 males and 144 females, selected using the convenience sampling method. The results showed that although the main purpose of the park-goers is not to use OGE, most of the participants thought that OGE use is not safe. Participants also reported that they had experienced various injuries while using OGE. Overall, although OGE seems to contribute to the healthy living needs of people, the relevant bodies may undertake improvements to increase their ergonomics and safety. In addition, it reveals the necessity of providing necessary trainings for the conscious use of OGE by users and having user quidebooks on OGE. Future research may reveal OGE-oriented behaviors and actual physical activity findings of OGE users. Finally, further research is encouraged to identify the health effects and safety-related issues of OGE.
\end{abstract}


Keywords: Physical activity, Outdoor gym, Gym areas, Injury

\section{Introduction}

In recent years, the world has been suffering from a pronounced elevation in sedentary lifestyle - accounting for most obesity cases and chronic conditions - which significantly increases the costs of healthcare services. Although the literature proves that people engaged in physical activities enjoy the benefits of healthy living (Nelson et al., 2007), the inability to engage in adequate physical activity still remains a persistent public health problem within larger populations (Haskell et al., 2007). According to the socio-ecological model (Sallis et al., 1998; McLeroyet et al., 1988), the human-made environment is a critical contributor to physical activity in large populations. So far, a wide range of studies have investigated the impact of the human-made environment on shaping the physical activity and health of the populations (Van Cauwenberg et al., 2011; Durand et al., 2011). Therefore, in terms of enabling physical activity among people, parks significantly contribute to reducing the disease burden in developed countries (Duncan et al., 2005).

Utilized as venues for recreation and sports (Rohde \& Kendle, 1997), parks close to residential areas often offer many opportunities for a range of activities, including those associated with physical activity (Cohen et al., 2014; Godbey \& Mowen, 2010). Consequently, parks are identified as ideal environments to promote physical activity through a range of physical, political, and pragmatic strategies (Bedimo-Rung et al., 2005; Bedimo-Rung et al., 2016). Previous research identified environmental factors of parks influencing visits and physical activities as ease of access, distance, locations, dimensions, amenities, social environment, organized activities, and nature orientation (Cohen et al., 2010; Lin et al., 2014). Two-way perceptions affect the use of parks and open spaces, namely safety and aesthetic appeal (Evenson et al., 2006).

It was previously revealed that people visiting parks engage in physical activities at various levels. For example, McKenzie et al. (2006) observed a total of 16,244 people in eight parks in Los Angeles and found that most visitors engaged in sedentary activities (such as lying down, sitting on benches, chatting with friends) and that such behaviors were prevalent in women (71\%) compared to men (62\%). They further investigated the existence of differences between the types of areas and the physical activity levels of visitors. The results revealed that vigorous physical activities were more likely performed in multi-purpose areas and less frequent in picnic areas (McKenzie et al., 2006).

Initial park-based physical activity research focused on general park features (e.g., distance, park size, park type, etc.) concerning physical activity instead of specific park features/opportunities. However, successor researchers scrutinized the contribution of certain park features and physical activity designs in these parks (Kaczynski et al., 2011; Cohen et al., 2015). Besenyi et al. (2013) conducted a study in four Kansas City parks and found that paved roads and tennis courts were active areas for adults while playgrounds were for children. Such a facility/activity feature contributed to significant increases in OGE installations in parks and other public spaces. 
OGE is exercise equipment similar to those found in traditional indoor gyms and rehabilitation clinics and often provides free access for people to exercise in outdoor settings. In general, an OGE area consists of several mechanical stations oriented to improve specific areas of a body (Aparicio, 2009). For example, the arm stretching station is designed to increase flexibility targeting the upper extremity area.

On the other hand, several studies showed that OGE poses many safety issues due to lack of oversight and inadequate instructions. A study reported that $79 \%$ of users experienced health problems after using OGE, and 54\% complained of muscle pain (Doğru et al., 2015). In addition, substantial research reported that many OGE accidents or sports injuries occurred due to misusing the equipment (Xie, 2012; Yan, 2016). Xie (2012) also reported that $66.5 \%$ of Chinese users were injured after using OGE. The main factors attributed to injuries were equipment malfunction (58.3\%) and misuse behavior (31.4\%) (Xie, 2012; Yan, 2016).

Many other studies carried out between 1970 and 1990 only considered playgrounds for children regarding park-related accidents or injuries. Accordingly, these studies reported many serious accidents resulting in fractures, concussions, head injuries, and prolonged hospital stays. In this respect, the previous research identified factors associated with playground injuries, including the playground environment, behaviors, and frequency of use. Some studies concluded that playground-related injuries were significantly reduced after generating playground safety instructions/guidelines (Mott et al., 1994; Laforest et al., 2001).

As OGE installation is a new feature in many regions, parks, or open spaces around the world, a limited number of studies have investigated the risks of such equipment based on their operating methods and models. Understanding how people use OGE in real settings may help identify issues leading to OGE-causing accidents and injuries. Therefore, we carried out this study to understand the OGE use behaviors of people considering ergonomics and safety of OGE. The specific objectives of this study were (1) to investigate whether there were differences between OGE users by gender and age and (2) to evaluate the potential risks of each OGE use behavior by sports and orthopedic disease history of the users.

\section{Method}

We carried out this descriptive study to uncover the actual situation on the subject (Gliner, Morgan, \& Leech, 2016) in 4 parks in Erenler district of Sakarya city. While the research universe consisted of individuals doing physical exercises in parks with OGE in Sakarya, the sample was composed of a total of 359 people, 215 males and 144 females, selected using the convenience sampling method.

\subsection{Data Collection}

We administered a questionnaire to the participants upon their voluntary consent between 06.30-10.30 a.m. and 5.30-9.30 p.m. in all months of the year in the 2020 .

\subsection{Data Analysis}

We presented demographic characteristics of the participants, including gender, age, and sports and orthopedic disorder history, and identified their relationships with the variables "injury 
after OGE use," "ergonomics of OGE," and "safety of OGE” using the Chi-square test.

\section{Results}

Table 1. Gender

\begin{tabular}{|l|l|l|}
\hline Gender & F & \% \\
\hline Female & 144 & 40.1 \\
\hline Male & 215 & 59.9 \\
\hline
\end{tabular}

As shown in Table 1, more than half of OGE users were males $(n=215 ; 59.9 \%)$, while the number of female users was 144 (40.1\%).

Table 2. Demographic characteristics of the participants

\begin{tabular}{|c|c|c|c|}
\hline Gender & Age & $\mathbf{F}$ & $\%$ \\
\hline \multirow{3}{*}{ Female } & $18-40$ years & 53 & 36.8 \\
\hline & 41-60 years & 65 & 45.1 \\
\hline & 61 years and over & 26 & 18.1 \\
\hline \multirow{3}{*}{ Male } & $18-40$ years & 55 & 25.6 \\
\hline & 41-60 years & 127 & 59.1 \\
\hline & 61 years and over & 33 & 15.3 \\
\hline Gender & Previous orthopedic disorder & $\mathbf{F}$ & $\%$ \\
\hline \multirow{2}{*}{ Female } & Yes & 17 & 11.8 \\
\hline & No & 127 & 88.2 \\
\hline \multirow{2}{*}{ Erkek } & Yes & 42 & 19.5 \\
\hline & No & 173 & 80.5 \\
\hline Gender & Sports history & $\mathbf{F}$ & $\%$ \\
\hline \multirow{2}{*}{ Female } & Yes & 14 & 9.7 \\
\hline & No & 130 & 90.3 \\
\hline \multirow{2}{*}{ Erkek } & Yes & 39 & 18.1 \\
\hline & No & 176 & 81.9 \\
\hline
\end{tabular}


Among OGE users, while the number of participants aged 61 years and over was the least, those aged 41-60 years had the highest participation rate. Although the frequency of previous orthopedic disorders was low, the female participants had experienced fewer disorders than males. We also discovered that the majority of participants did not have a sports history.

Table 3. Ergonomics and safety of OGE use by gender

\begin{tabular}{|l|l|l|l|}
\hline & \multicolumn{2}{|c|}{ Do you think OGE use is ergonomic? } & \\
\hline Gender & Yes & No & p \\
\hline Female & $16(11.1 \%)$ & $128(88.9 \%)$ & .588 \\
\hline Male & $28(13.0 \%)$ & $(87.0 \%)$ & \\
\hline & \multicolumn{2}{|c|}{ Do you think OGE use is safe? } & \\
\hline Gender & Yes & No & p \\
\hline Female & $29(20.1 \%)$ & $115(79.9 \%)$ & .802 \\
\hline Male & $41(19.1 \%)$ & $174(80.9 \%)$ & \\
\hline
\end{tabular}

Table 2 shows that the majority of the participants did not think OGE use is ergonomic and safe. Yet, we could not find significant differences between the participants' perceptions of ergonomics and safety of OGE use by gender $(p>0.05)$.

Table 4. Ergonomics and safety of OGE use by sports history

\begin{tabular}{|l|l|l|l|}
\hline & \multicolumn{2}{|c|}{ Do you think OGE use is ergonomic? } & \\
\hline Sports history & Yes & No & p \\
\hline Yes & $15(28.3 \%)$ & $38(71.7 \%)$ & .000 \\
\hline No & $29(9.5 \%)$ & $277(90.5 \%)$ & \\
\hline Sports history & \multicolumn{2}{|c|}{ Do you think OGE use is safe? } & \\
\hline Yes & Yes & No & p \\
\hline No & $16(30.2 \%)$ & $37(69.8 \%)$ & \multirow{2}{*}{.033} \\
\hline
\end{tabular}

As shown in Table 4, we found that those with sports history thought OGE to be ergonomic 


\section{Macrothink Mnstitute"'}

significantly more than those without sports history. Similarly, the participants with a sports background found OGE to be safe significantly more than their counterparts. Accordingly, the participants' perceptions of ergonomics and safety of OGE use significantly differed by sports history $(\mathrm{p}<0.05)$.

Table 5. Ergonomics and safety of OGE use by orthopedic disorder history

\begin{tabular}{|l|l|l|l|}
\hline & \multicolumn{2}{|l|}{ Do you think OGE use is ergonomic? } & \multirow{2}{*}{} \\
\hline Orthopedic disorder history & Yes & No & p \\
\hline Yes & $14(23.7 \%)$ & $45(76.3 \%)$ & \multirow{2}{*}{.003} \\
\hline No & $30(10.0 \%)$ & $270(90.0 \%)$ & \\
\hline & Do you think OGE use is safe? & No & \multirow{2}{*}{.859} \\
\hline Orthopedic disorder history & Yes & $47(79.7 \%)$ & \\
\hline Yes & $12(20.3 \%)$ & $242(80.7 \%)$ & \\
\hline No & $58(19.3 \%)$ & & \\
\hline
\end{tabular}

We found that the participants' perceptions of ergonomics of OGE significantly differed by their orthopedic disorder history. Interestingly, the participants with orthopedic disorder history thought OGE to be ergonomic significantly more than those without such a medical experience $(\mathrm{p}<0.05)$. On the other hand, the majority of the participants, those both with and without orthopedic disorder history, considered OGE not to be safe. There were no significant differences in their safety perceptions regarding OGE by their medical history $(p>0.05)$. 
Table 6. Ergonomics and safety of OGE use by age

\begin{tabular}{|c|c|c|c|}
\hline & \multicolumn{2}{|c|}{ Do you think OGE use is ergonomic? } & \\
\hline Age & Yes & No & $\mathrm{p}$ \\
\hline $18-40$ years & $9(8.3 \%)$ & $99(91.7 \%)$ & \multirow{3}{*}{0.150} \\
\hline $41-60$ years & $24(12.5 \%)$ & $168(87.5 \%)$ & \\
\hline \multirow[t]{2}{*}{61 years and over } & $11(18.6 \%)$ & $48(81.4 \%)$ & \\
\hline & \multicolumn{2}{|c|}{ Do you think OGE use is safe? } & \\
\hline Age & Yes & No & $\mathrm{p}$ \\
\hline $18-40$ years & $16(14.8 \%)$ & $92(85.2 \%)$ & \multirow{3}{*}{0.233} \\
\hline $41-60$ years & $39(20.3 \%)$ & $153(79.7 \%)$ & \\
\hline 61 years and over & $15(25.4 \%)$ & $44(74.6 \%)$ & \\
\hline
\end{tabular}

Table 6 presents that all age groups mostly did not find OGE use is ergonomic and safe. We could not find any significant differences in the participants' perceptions of ergonomics and safety of OGE use by age $(\mathrm{p}>0.05)$. 
Table 7. Injuries from OGE by gender

\begin{tabular}{|c|c|c|c|c|c|}
\hline OGE & Gender & Injury & Strain & None & $\mathrm{p}$ \\
\hline \multirow{2}{*}{$\begin{array}{l}\text { Have you had any injury or severe } \\
\text { strain on the leg stretching station? }\end{array}$} & Female & $19(13.2 \%)$ & $98(68.1 \%)$ & $2(18.8 \%)$ & \multirow{2}{*}{$.010 *$} \\
\hline & Male & $57(26.5 \%)$ & $122(56.7 \%)$ & $36(16.7 \%)$ & \\
\hline \multirow{2}{*}{$\begin{array}{l}\text { Have you had any injury or severe } \\
\text { strain on leg lift and fitness stations? }\end{array}$} & Female & $10(6.9 \%)$ & $101(70.1 \%)$ & $33(22.9 \%)$ & \multirow{2}{*}{$.001 *$} \\
\hline & Male & $44(20.5 \%)$ & $120(55.8 \%)$ & $51(23.7 \%)$ & \\
\hline \multirow{2}{*}{$\begin{array}{l}\text { Have you had any injury or severe } \\
\text { strain on the leg stretching station (2)? }\end{array}$} & Female & $18(12.5 \%)$ & $99(68.8 \%)$ & $27(18.8 \%)$ & \multirow{2}{*}{.612} \\
\hline & Male & $35(16.3 \%)$ & $142(66.0 \%)$ & $38(17.7 \%)$ & \\
\hline \multirow{2}{*}{$\begin{array}{l}\text { Have you had any injury or severe } \\
\text { strain on the hip twister? }\end{array}$} & Female & $17(11.8 \%)$ & $96(66.7 \%)$ & $31(21.5)$ & \multirow{2}{*}{$.000 *$} \\
\hline & Male & $74(34.4 \%)$ & $123(57.2 \%)$ & $18(8.4 \%)$ & \\
\hline \multirow{2}{*}{$\begin{array}{l}\text { Have you had any injury or severe } \\
\text { strain on the cycling station? }\end{array}$} & Female & $17(11.8 \%)$ & $87(60.4 \%)$ & $40(27.8 \%)$ & \multirow{2}{*}{$.000^{*}$} \\
\hline & Male & $52(24.2 \%)$ & $133(61.9 \%)$ & $30(14.0 \%)$ & \\
\hline \multirow{2}{*}{$\begin{array}{l}\text { Have you had any injury or severe } \\
\text { strain on the double walking station? }\end{array}$} & Female & $23(16.0 \%)$ & $76(52.8 \%)$ & $45(31.3 \%)$ & \multirow{2}{*}{$.005^{*}$} \\
\hline & Male & $37(17.2 \%)$ & $142(66.0 \%)$ & $36(16.7 \%)$ & \\
\hline \multirow{2}{*}{$\begin{array}{l}\text { Have you had any injury or severe } \\
\text { strain on the trio fitness station? }\end{array}$} & Female & $10(6.9 \%)$ & $90(62.5 \%)$ & $44(30.6 \%)$ & \multirow{2}{*}{$.005^{*}$} \\
\hline & Male & $37(17.2 \%)$ & $134(62.3 \%)$ & $44(20.5 \%)$ & \\
\hline \multirow{2}{*}{$\begin{array}{l}\text { Have you had any injury or severe } \\
\text { strain on the rider station? }\end{array}$} & Female & $10(6.9 \%)$ & $88(61.1 \%)$ & $46(31.9 \%)$ & \multirow{2}{*}{$.010 *$} \\
\hline & Male & $17(7.9 \%)$ & $159(74.0 \%)$ & $39(18.1 \%)$ & \\
\hline \multirow{2}{*}{$\begin{array}{l}\text { Have you had any injury or severe } \\
\text { strain on the t'ai chi spinner? }\end{array}$} & Female & $12(8.3 \%)$ & $96(66.7 \%)$ & $36(25.0 \%)$ & \multirow{2}{*}{.733} \\
\hline & Male & $23(10.7 \%)$ & $137(63.7 \%)$ & $55(25.0 \%)$ & \\
\hline \multirow{2}{*}{$\begin{array}{l}\text { Have you had any injury or severe } \\
\text { strain on the back massage station? }\end{array}$} & Female & $18(12.5 \%)$ & $76(52.8 \%)$ & $50(34.7 \%)$ & \multirow{2}{*}{$.003 *$} \\
\hline & Male & $49(22.8 \%)$ & $77(35.8 \%)$ & $89(41.4 \%)$ & \\
\hline \multirow{2}{*}{$\begin{array}{l}\text { Have you had any injury or severe } \\
\text { strain on the pushup and crunch station? }\end{array}$} & Female & $26(18.1 \%)$ & $80(55.6 \%)$ & $38(26.4 \%)$ & \multirow{2}{*}{$.000 *$} \\
\hline & Male & $48(22.3 \%)$ & $60(27.9 \%)$ & $107(49.8 \%)$ & \\
\hline \multirow{2}{*}{$\begin{array}{l}\text { Have you had any injury or severe } \\
\text { strain on the space walker station? }\end{array}$} & Female & $35(24.3 \%)$ & $61(42.4 \%)$ & $48(33.3 \%)$ & \multirow{2}{*}{.132} \\
\hline & Male & $39(18.1 \%)$ & $83(38.6 \%)$ & $93(43.3 \%)$ & \\
\hline \multirow{2}{*}{$\begin{array}{l}\text { Have you had any injury or severe } \\
\text { strain on the air skier? }\end{array}$} & Female & $36(25.0 \%)$ & $64(44.4 \%)$ & $44(30.6 \%)$ & \multirow{2}{*}{.258} \\
\hline & Male & $41(19.1 \%)$ & $113(52.6 \%)$ & $61(28.4 \%)$ & \\
\hline
\end{tabular}

We found that the participants experienced injuries or strains from many stations while doing sports, which differed significantly by gender $(\mathrm{p}<0.05)$. Yet, there were no significant differences in injuries and strains from the leg stretching station (2), t'ai chi spinner, space walker station, and air skier by gender $(p>0.05)$. 
Table 8. Injuries from OGE by orthopedic disorder history

\begin{tabular}{|c|c|c|c|c|c|}
\hline OGE & $\begin{array}{l}\text { Orthopedic } \\
\text { disorder history }\end{array}$ & Injury & Strain & None & $\mathrm{p}$ \\
\hline \multirow{2}{*}{$\begin{array}{l}\text { Have you had any injury or severe } \\
\text { strain on the leg stretching station? }\end{array}$} & Yes & $16(27.1 \%)$ & $26(44.1 \%)$ & $17(28.8 \%)$ & \multirow{2}{*}{$.008^{*}$} \\
\hline & No & $60(20.0 \%)$ & $194(64.7 \%)$ & $46(15.3 \%)$ & \\
\hline \multirow{2}{*}{$\begin{array}{l}\text { Have you had any injury or severe } \\
\text { strain on leg lift and fitness stations? }\end{array}$} & Yes & $17(28.8 \%)$ & $25(42.4 \%)$ & $17(28.8 \%)$ & \multirow{2}{*}{$.001 *$} \\
\hline & No & $37(12.3 \%)$ & $196(65.3 \%)$ & $67(22.3 \%)$ & \\
\hline \multirow{2}{*}{$\begin{array}{l}\text { Have you had any injury or severe } \\
\text { strain on the leg stretching station (2)? }\end{array}$} & Yes & $10(16.9 \%)$ & $42(71.2 \%)$ & $7(11.9 \%)$ & \multirow{2}{*}{.383} \\
\hline & No & $43(14.3 \%)$ & $199(66.3 \%)$ & $58(19.3 \%)$ & \\
\hline \multirow{2}{*}{$\begin{array}{l}\text { Have you had any injury or severe } \\
\text { strain on the hip twister? }\end{array}$} & Yes & $34(57.6 \%)$ & $21(35.6 \%)$ & $4(6.8 \%)$ & \multirow{2}{*}{$.000^{*}$} \\
\hline & No & $57(19.0 \%)$ & $198(66.0 \%)$ & $45(15.0 \%)$ & \\
\hline \multirow{2}{*}{$\begin{array}{l}\text { Have you had any injury or severe } \\
\text { strain on the cycling station? }\end{array}$} & Yes & $23(39.0 \%)$ & $23(39.0 \%)$ & $13(22.0 \%)$ & \multirow{2}{*}{$.000 *$} \\
\hline & No & $46(15.3 \%)$ & $197(65.7 \%)$ & $57(19.0 \%)$ & \\
\hline \multirow{2}{*}{$\begin{array}{l}\text { Have you had any injury or severe } \\
\text { strain on the double walking station? }\end{array}$} & Yes & $18(30.5 \%)$ & $28(47.5 \%)$ & $13(22.0 \%)$ & \multirow{2}{*}{$.006^{*}$} \\
\hline & No & $42(17.0 \%)$ & $190(63.3 \%)$ & $68(22.7 \%)$ & \\
\hline \multirow{2}{*}{$\begin{array}{l}\text { Have you had any injury or severe } \\
\text { strain on the trio fitness station? }\end{array}$} & Yes & $14(23.7 \%)$ & $30(50.8 \%)$ & $15(25.4 \%)$ & \multirow{2}{*}{$.022 *$} \\
\hline & No & $33(11.0 \%)$ & $194(64.7 \%)$ & $73(24.3 \%)$ & \\
\hline \multirow{2}{*}{$\begin{array}{l}\text { Have you had any injury or severe } \\
\text { strain on the rider station? }\end{array}$} & Yes & $7(11.9 \%)$ & $41(69.5 \%)$ & $11(18.6 \%)$ & \multirow{2}{*}{.282} \\
\hline & No & $20(6.7 \%)$ & $206(68.7 \%)$ & $74(24.7 \%)$ & \\
\hline \multirow{2}{*}{$\begin{array}{l}\text { Have you had any injury or severe } \\
\text { strain on the t'ai chi spinner? }\end{array}$} & Yes & $7(11.9 \%)$ & $23(39.0 \%)$ & $29(49.2 \%)$ & \multirow{2}{*}{$.000^{*}$} \\
\hline & No & $28(9.3 \%)$ & $210(70.0 \%)$ & $62(20.7 \%)$ & \\
\hline \multirow{2}{*}{$\begin{array}{l}\text { Have you had any injury or severe } \\
\text { strain on the back massage station? }\end{array}$} & Yes & $22(37.3 \%)$ & $16(27.1 \%)$ & $21(35.6 \%)$ & \multirow{2}{*}{$.000 *$} \\
\hline & No & $45(15.0 \%)$ & $137(45.7 \%)$ & $118(39.3 \%)$ & \\
\hline \multirow{2}{*}{$\begin{array}{l}\text { Have you had any injury or severe } \\
\text { strain on the pushup and crunch station? }\end{array}$} & Yes & $23(39.0 \%)$ & $8(13.6 \%)$ & $28(47.5 \%)$ & \multirow{2}{*}{$.000 *$} \\
\hline & No & $51(17.0 \%)$ & $132(44.0 \%)$ & $117(39.0 \%)$ & \\
\hline \multirow{2}{*}{$\begin{array}{l}\text { Have you had any injury or severe } \\
\text { strain on the space walker station? }\end{array}$} & Yes & $22(37.3 \%)$ & $10(16.9 \%)$ & $27(45.8 \%)$ & \multirow{2}{*}{$.000^{*}$} \\
\hline & No & $52(17.3 \%)$ & $134(44.7 \%)$ & $114(38.0 \%)$ & \\
\hline \multirow{2}{*}{$\begin{array}{l}\text { Have you had any injury or severe } \\
\text { strain on the air skier? }\end{array}$} & Yes & $22(37.3 \%)$ & $19(32.2 \%)$ & $18(30.5 \%)$ & \multirow{2}{*}{$.002 *$} \\
\hline & No & $55(18.3 \%)$ & $158(52.7 \%)$ & $87(29.0 \%)$ & \\
\hline
\end{tabular}

As shown in Table 8, the participants experienced injuries or strains from many stations while doing sports, which differed significantly by orthopedic disorder history $(p<0.05)$. However, it was not the case for injuries and strains from the leg stretching station (2) and rider station $(\mathrm{p}<$ $0.05)$. 
Table 9. Injuries from OGE by sports history

\begin{tabular}{|c|c|c|c|c|c|}
\hline OGE & Sports history & Injury & Strain & None & $\mathrm{p}$ \\
\hline \multirow{2}{*}{$\begin{array}{l}\text { Have you had any injury or severe } \\
\text { strain on the leg stretching station? }\end{array}$} & Yes & $10(18.9 \%)$ & $24(45.3 \%)$ & $19(35.8 \%)$ & \multirow{2}{*}{.001} \\
\hline & No & $66(21.6 \%)$ & $196(64.1 \%)$ & $44(14.4 \%)$ & \\
\hline \multirow{2}{*}{$\begin{array}{l}\text { Have you had any injury or severe } \\
\text { strain on leg lift and fitness stations? }\end{array}$} & Yes & $3(5.7 \%)$ & $28(52.8 \%)$ & $22(41.5 \%)$ & \multirow{2}{*}{.001} \\
\hline & No & $51(16.7 \%)$ & $193(63.1 \%)$ & $62(20.3 \%)$ & \\
\hline \multirow{2}{*}{$\begin{array}{l}\text { Have you had any injury or severe } \\
\text { strain on the leg stretching station (2)? }\end{array}$} & Yes & $3(5.7 \%)$ & $39(73.6 \%)$ & $11(20.8 \%)$ & \multirow{2}{*}{$.128 *$} \\
\hline & No & $50(16.3 \%)$ & $202(66.0 \%)$ & $54(17.6 \%)$ & \\
\hline \multirow{2}{*}{$\begin{array}{l}\text { Have you had any injury or severe } \\
\text { strain on the hip twister? }\end{array}$} & Yes & $24(45.3 \%)$ & $22(41.5 \%)$ & $7(13.2 \%)$ & \multirow{2}{*}{.001} \\
\hline & No & $67(21.9 \%)$ & $197(64.4 \%)$ & $42(13.7 \%)$ & \\
\hline \multirow{2}{*}{$\begin{array}{l}\text { Have you had any injury or severe } \\
\text { strain on the cycling station? }\end{array}$} & Yes & $6(11.3 \%)$ & $29(54.7 \%)$ & $18(34.0 \%)$ & \multirow{2}{*}{.010} \\
\hline & No & $63(20.6 \%)$ & $191(62.4 \%)$ & $52(17.0 \%)$ & \\
\hline \multirow{2}{*}{$\begin{array}{l}\text { Have you had any injury or severe } \\
\text { strain on the double walking station? }\end{array}$} & Yes & $6(11.3 \%)$ & $20(37.7 \%)$ & $27(50.9 \%)$ & \multirow{2}{*}{.000} \\
\hline & No & $54(17.6 \%)$ & $198(64.7 \%)$ & $54(17.6 \%)$ & \\
\hline \multirow{2}{*}{$\begin{array}{l}\text { Have you had any injury or severe } \\
\text { strain on the trio fitness station? }\end{array}$} & Yes & $12(22.6 \%)$ & $24(45.3 \%)$ & $17(32.1 \%)$ & \multirow{2}{*}{.013} \\
\hline & No & $35(11.4 \%)$ & $200(65.4 \%)$ & $71(23.2 \%)$ & \\
\hline \multirow{2}{*}{$\begin{array}{l}\text { Have you had any injury or severe } \\
\text { strain on the rider station? }\end{array}$} & Yes & $3(5.7 \%)$ & $38(71.7 \%)$ & $12(22.6 \%)$ & \multirow{2}{*}{$.823^{*}$} \\
\hline & No & $24(7.8 \%)$ & $209(68.3 \%)$ & $73(23.9 \%)$ & \\
\hline \multirow{2}{*}{$\begin{array}{l}\text { Have you had any injury or severe } \\
\text { strain on the t'ai chi spinner? }\end{array}$} & Yes & $4(7.5 \%)$ & $20(37.7 \%)$ & $29(54.7 \%)$ & \multirow{2}{*}{.000} \\
\hline & No & $31(10.1 \%)$ & $213(69.6 \%)$ & $62(20.3 \%)$ & \\
\hline \multirow{2}{*}{$\begin{array}{l}\text { Have you had any injury or severe } \\
\text { strain on the back massage station? }\end{array}$} & Yes & $22(41.5 \%)$ & $17(32.1 \%)$ & $14(26.4 \%)$ & \multirow{2}{*}{.000} \\
\hline & No & $45(14.7 \%)$ & $136(44.4 \%)$ & $125(40.8 \%)$ & \\
\hline \multirow{2}{*}{$\begin{array}{l}\text { Have you had any injury or severe } \\
\text { strain on the pushup and crunch station? }\end{array}$} & Yes & $7(13.2 \%)$ & $13(24.5 \%)$ & $33(62.3 \%)$ & \multirow{2}{*}{.002} \\
\hline & No & $67(21.9 \%)$ & $127(41.5 \%)$ & $112(36.6 \%)$ & \\
\hline \multirow{2}{*}{$\begin{array}{l}\text { Have you had any injury or severe } \\
\text { strain on the space walker station? }\end{array}$} & Yes & $11(20.8 \%)$ & $14(26.4 \%)$ & $28(52.8 \%)$ & \multirow{2}{*}{$.055^{*}$} \\
\hline & No & $63(20.6 \%)$ & $130(42.5 \%)$ & $113(36.9 \%)$ & \\
\hline \multirow{2}{*}{$\begin{array}{l}\text { Have you had any injury or severe } \\
\text { strain on the air skier? }\end{array}$} & Yes & $11(20.8 \%)$ & $32(60.4 \%)$ & $10(18.9 \%)$ & \multirow{2}{*}{$.146^{*}$} \\
\hline & No & $66(21.6 \%)$ & $145(47.4 \%)$ & $95(31.0 \%)$ & \\
\hline
\end{tabular}

Those both with and without sports history reported injuries and strains from various stations 
while doing sports, and experiencing such undesirable experiences on outdoor sports equipment differed significantly by sports history $(p<0.05)$. Nevertheless, there were no significant differences in injuries and strains from the leg stretching station (2), rider station, space walker station, and air skier $(\mathrm{p}>0.05)$.

Table 10. Injuries from OGE by age

\begin{tabular}{|c|c|c|c|c|c|}
\hline OGE & Age & Injury & Strain & None & $\mathrm{p}$ \\
\hline \multirow{3}{*}{$\begin{array}{l}\text { Have you had any injury or severe } \\
\text { strain on the leg stretching station? }\end{array}$} & $18-40$ years & $11(10.2 \%)$ & $76(70.4 \%)$ & $21(19.4 \%)$ & \multirow{3}{*}{.000} \\
\hline & $41-60$ years & $41(21.4 \%)$ & $119(62.0 \%)$ & $32(16.7 \%)$ & \\
\hline & 61 years and over & $24(40.7 \%)$ & $25(42.4 \%)$ & $10(16.9 \%)$ & \\
\hline \multirow{3}{*}{$\begin{array}{l}\text { Have you had any injury or severe } \\
\text { strain on leg lift and fitness stations? }\end{array}$} & $18-40$ years & $10(9.3 \%)$ & $64(59.3 \%)$ & $34(31.5 \%)$ & \multirow{3}{*}{.000} \\
\hline & $41-60$ years & $22(11.5 \%)$ & $131(68.2 \%)$ & $39(20.3 \%)$ & \\
\hline & 61 years and over & $22(37.3 \%)$ & $26(44.1 \%)$ & $11(18.6 \%)$ & \\
\hline \multirow{3}{*}{$\begin{array}{l}\text { Have you had any injury or severe } \\
\text { strain on the leg stretching station (2)? }\end{array}$} & $18-40$ years & $9(8.3 \%)$ & $74(68.5 \%)$ & $25(23.1 \%)$ & \multirow{3}{*}{.000} \\
\hline & $41-60$ years & $21(10.9 \%)$ & $134(69.8 \%)$ & $37(19.3 \%)$ & \\
\hline & 61 years and over & $23(39.0 \%)$ & $33(55.9 \%)$ & $3(5.1 \%)$ & \\
\hline \multirow{3}{*}{$\begin{array}{l}\text { Have you had any injury or severe } \\
\text { strain on the hip twister? }\end{array}$} & $18-40$ years & $13(12.0 \%)$ & $72(66.7 \%)$ & $23(21.3 \%)$ & \multirow{3}{*}{.000} \\
\hline & $41-60$ years & $44(22.9 \%)$ & $125(65.1 \%)$ & $23(12.0 \%)$ & \\
\hline & 61 years and over & $34(57.6 \%)$ & $22(37.3 \%)$ & $3(5.1 \%)$ & \\
\hline \multirow{3}{*}{$\begin{array}{l}\text { Have you had any injury or severe } \\
\text { strain on the cycling station? }\end{array}$} & $18-40$ years & $14(13.0 \%)$ & $70(64.8 \%)$ & $24(22.2 \%)$ & \multirow{3}{*}{.000} \\
\hline & 41-60 years & $28(14.6 \%)$ & $133(69.3 \%)$ & $31(16.1 \%)$ & \\
\hline & 61 years and over & $27(45.8 \%)$ & $17(28.8 \%)$ & $15(25.4 \%)$ & \\
\hline \multirow{3}{*}{$\begin{array}{l}\text { Have you had any injury or severe } \\
\text { strain on the double walking station? }\end{array}$} & $18-40$ years & $12(11.1 \%)$ & $70(64.8 \%)$ & $26(24.1 \%)$ & \multirow{3}{*}{.000} \\
\hline & 41-60 years & $28(14.6 \%)$ & $126(65.6 \%)$ & $38(19.8 \%)$ & \\
\hline & 61 years and over & $20(33.9 \%)$ & $22(37.3 \%)$ & $17(28.8 \%)$ & \\
\hline \multirow{3}{*}{$\begin{array}{l}\text { Have you had any injury or severe } \\
\text { strain on the trio fitness station? }\end{array}$} & $18-40$ years & $6(5.6 \%)$ & $67(62.0 \%)$ & $35(32.4 \%)$ & \multirow{3}{*}{.000} \\
\hline & 41-60 years & $26(13.5 \%)$ & $132(68.8 \%)$ & $34(17.7 \%)$ & \\
\hline & 61 years and over & $15(25.4 \%)$ & $25(42.4 \%)$ & $19(32.2 \%)$ & \\
\hline \multirow{3}{*}{$\begin{array}{l}\text { Have you had any injury or severe } \\
\text { strain on the rider station? }\end{array}$} & $18-40$ years & $2(1.9 \%)$ & $83(76.9 \%)$ & $23(21.3 \%)$ & \multirow{3}{*}{.000} \\
\hline & $41-60$ years & $13(6.8 \%)$ & $123(64.1 \%)$ & $56(29.2 \%)$ & \\
\hline & 61 years and over & $12(20.3 \%)$ & $41(69.5 \%)$ & $6(10.2 \%)$ & \\
\hline
\end{tabular}




\begin{tabular}{|c|c|c|c|c|c|}
\hline \multirow{3}{*}{$\begin{array}{l}\text { Have you had any injury or severe } \\
\text { strain on the t'ai chi spinner? }\end{array}$} & $18-40$ years & $6(5.6 \%)$ & $76(70.4 \%)$ & $26(24.1 \%)$ & \multirow{3}{*}{.303} \\
\hline & $41-60$ years & $20(10.4 \%)$ & $121(63.0 \%)$ & $51(26.6 \%)$ & \\
\hline & 61 years and over & $9(15.3 \%)$ & $36(61.0 \%)$ & $14(23.7 \%)$ & \\
\hline \multirow{3}{*}{$\begin{array}{l}\text { Have you had any injury or severe } \\
\text { strain on the back massage station? }\end{array}$} & $18-40$ years & $9(8.3 \%)$ & $63(58.3 \%)$ & $36(33.3 \%)$ & \multirow{3}{*}{.000} \\
\hline & $41-60$ years & $39(20.3 \%)$ & $66(34.4 \%)$ & $87(45.3 \%)$ & \\
\hline & 61 years and over & $19(32.2 \%)$ & $24(40.7 \%)$ & $16(27.1 \%)$ & \\
\hline \multirow{3}{*}{$\begin{array}{l}\text { Have you had any injury or severe } \\
\text { strain on the pushup and crunch station? }\end{array}$} & $18-40$ years & $22(20.4 \%)$ & $43(39.8 \%)$ & $43(39.8 \%)$ & \multirow{3}{*}{.532} \\
\hline & $41-60$ years & $35(18.2 \%)$ & $77(40.1 \%)$ & $80(41.7 \%)$ & \\
\hline & 61 years and over & $17(28.8 \%)$ & $20(33.9 \%)$ & $22(37.3 \%)$ & \\
\hline \multirow{3}{*}{$\begin{array}{l}\text { Have you had any injury or severe } \\
\text { strain on the space walker station? }\end{array}$} & $18-40$ years & $17(15.7 \%)$ & $46(42.6 \%)$ & $45(41.7 \%)$ & \multirow{3}{*}{.158} \\
\hline & $41-60$ years & $44(22.9 \%)$ & $81(42.2 \%)$ & $67(34.9 \%)$ & \\
\hline & 61 years and over & $13(22.0 \%)$ & $17(28.8 \%)$ & $29(49.2 \%)$ & \\
\hline \multirow{3}{*}{$\begin{array}{l}\text { Have you had any injury or severe } \\
\text { strain on the air skier? }\end{array}$} & $18-40$ years & $17(15.7 \%)$ & $46(42.6 \%)$ & $45(41.7 \%)$ & \multirow{3}{*}{.004} \\
\hline & $41-60$ years & $45(23.4 \%)$ & $106(55.2 \%)$ & $41(21.4 \%)$ & \\
\hline & 61 years and over & $15(25.4 \%)$ & $25(42.4 \%)$ & $19(32.2 \%)$ & \\
\hline
\end{tabular}

Injuries and strains from OGE were reported more by the participants aged 18-40 years and 41-60 years. In the age group of 61 years and over, the rates of those having experienced injuries and strains from most of the stations were close to each other. Accordingly, we found significant differences in injuries and strains from OGE by age $(p<0.05)$. Nonetheless, we could not reach any significant differences in injuries and strains from the t'ai chi spinner, pushup and crunch station, and space walker station $(\mathrm{p}>0.05)$.

\section{Discussion}

The OGE installation in public areas has become increasingly popular, as doing physical activity may prevent various health problems, and, therefore, OGE is an essential means for doing physical activity (Sibson et al., 2018; Madren, 2013; Kershaw et al., 2017). Yet, there is a lack of research interest in exploring the actual use of OGE regarding ergonomics and safety, although it may provide crucial information for evaluating the effectiveness of OGE and developing future park or open space initiatives. The results of this study, investigating ergonomic and safety experiences of OGE users, revealed that the participants exhibited a variety of behaviors while using the same OGE. A previous interview study showed that many users simply imitate how others use the equipment, as there are no information sessions or instructions on how to use them (Chow, 2013). Besides, equipment design may not restrict users to specific approaches to OGE use, resulting in potential injuries due to several non-indicative risky user behaviors. Accordingly, we found that those with sports history 
could change their behaviors when using OGE, which may be because such OGE users find their previous sports postures/patterns/movements pose ergonomics and safety gaps on the stations. They may also want to increase the severity of using OFE or explore different behaviors on the stations just for fun. The results are in line with previous research showing that users only use OGE for short periods of time (Chow et al., 2017).

In terms of demographic characteristics of the participants, the majority of OGE users were males $(n=215 ; 59.9 \%)$. While the number of those aged 61 years and over was the lowest in the study, most of the participants were between 41-60 years. Although it was generally below the average in this study, the male participants had had more orthopedic disorders than the females. Finally, we found that the majority of the participants did not have a sports background; the females had more sports experience before than the males.

We found that the participants' perceptions of ergonomics of OGE significantly differed by their orthopedic disorder history. Interestingly, the participants with orthopedic disorder history thought OGE to be ergonomic significantly more than those without such a medical experience $(p<0.05)$. On the other hand, the majority of the participants, those both with and without orthopedic disorder history, considered OGE not to be safe. There were no significant differences in their safety perceptions of OGE by their medical history $(p>0.05)$.

We found that all age groups mostly did not find OGE use is ergonomic and safe. We could not find any significant differences in the participants' perceptions of ergonomics and safety of OGE use by age ( $p>0.05)$. Similarly, the majority of the participants did not think OGE use is ergonomic and safe. Yet, we could not find significant differences between the participants' perceptions of ergonomics and safety of OGE use by gender $(p>0.05)$

In the literature, Cranney (2016) used data from observations and interviews. In the observational data, the most commonly used OGE were (1) pull-down, (2) elliptical trainer, (3) aerobic cycle, and (4) parallel bars. However, given the differences in available OGE between studies, it may be difficult to compare the findings. Another reason for the inability to compare the results can be attributed to the preferences of different activity types preferred by visitors from different backgrounds such as ethnicity, culture (Humpel et al., 2002; Onge \& Krueger, 2011).

Correct and proper OGE use is important to prevent injuries or other health problems. In the interviews, we encountered many OGE users to have been injured before. However, the focus should always be on improper OGE use behaviors as they may not immediately lead to acute sports injuries or serious accidents. Previous studies showed that many OGE areas lacked instruction signs, or users ignored the instructions provided, which can lead to accidents (Chow et al., 2017; Chow, 2013). However, beyond the improper OGE use, we found that our participants did not find OGE to be ergonomic and safe whether they had a sports history or not. Improper use or negative ergonomics and safety perceptions may hinder people from obtaining full benefits from OGE. Various chronic or acute injuries resulting from improper OGE use may limit or prevent doing physical activity, resulting in poor health.

The results showed that most OGE users in the parks where we collected the data were adults 
and young adults, respectively, consistent with the studies by Cohen et al. (2012) and Bettencourt and Neves (2012). Yet, the study by Cranney et al. (2016) showed that the majority of OGE users were older adults. One explanation for the discrepancy between their study and our research may be differences in ethnicity, culture, or environmental issues in the countries where the studies were carried out (Humpel et al., 2002; Onge \& Krueger, 2011).

As the previous research suggests, park visitors may walk, cycle, or do group exercises when visiting parks (Mora, 2012; Chow, 2013). Therefore, OGE is not the main source of physical activity for park visitors but often serves as an additional feature to increase park-based physical activity levels among people.

\section{Conclusion}

The OGE installation in public spaces has become increasingly popular to encourage people to be more physically active and more socially connected. However, this study provides empirical evidence for ergonomics and safety issues of OGE that may lead to accidents and injuries, which can lead to various liability issues for manufacturers or relevant authorities. The results suggest that it is essential for manufacturers to provide clear equipment operation manuals (or instruction videos) regarding the proper use of their equipment and warning messages about risky behaviors, as well as to improve their designs considering ergonomics and safety of their available models. Moreover, governments or local authorities that authorize or sponsor the OGE installations may hold training sessions where professional trainers can explain how to use OGE properly, safely, and effectively to satisfy each individual's ability and fitness level. This is especially necessary for older adults who may feel insecure when using OGE. Such informative sessions should also target parents with children, emphasizing possible risks to children in adult-only OGE areas. These sessions may also serve as marketing strategies to attract park visitors or promote new OGE areas.

Two limitations of the study should inevitably be noted. First, each OGE addressed in the focus interviews could not be objectively measured by experts regarding ergonomics and safety in a controlled laboratory setting. Secondly, we carried out this study to reveal how people use OGE in four parks in Sakarya city and whether OGE poses ergonomic and safety hazards. Therefore, our results may not be fully generalizable to other equipment types or regions. Despite these limitations, the findings have important implications for future research. For example, governments can formulate clear policies and regulations on safety issues before building fitness zones or OGE installations. Moreover, manufacturers may design and develop equipment that meets certain safety, ergonomics, and sustainability principles. Park officials may hold sessions in the community to share user guidelines of OGE with the public and implement effective outreach strategies. In terms of visibility, governments may attach tags to equipment with a quick response code that links to a video showing how to use each OGE properly. Finally, park officials may set up warning signs to restrict children's use of OGE and routinely monitor and manage OGE areas.

Overall, this study raised many questions that may attract the attention of future research. Accordingly, future studies may explore other OGE types and user behaviors and identify whether different OGE designs are ergonomic and safe. Also, future research may thoroughly 
investigate current and previous OGE users for their experience of injury/accident and identify certain behaviors associated with these accidents. Another study may invite participants to perform the different OGE behaviors observed in this study and use electromyography to detect the activation of the muscles involved to clearly demonstrate the impact of different behaviors on the human body and to identify potential risks or benefits inherent in each station. In any case, while promoting an active lifestyle and public health, ensuring the safety of OGE users should be a priority.

\section{Acknowledgements}

Identify grants or other financial support (and the source, if appropriate) for your study; do not precede grant numbers by No. or \#. Next, acknowledge colleagues who assisted in conducting the study or critiquing the manuscript. Do not acknowledge the persons routinely involved in the review and acceptance of manuscripts - peer reviewers or editors, associate editors, and consulting editors of the journal in which the article is to appear. In this paragraph, also explain any special agreements concerning authorship, such as if authors contributed equally to the study. End this paragraph with thanks for personal assistance, such as in manuscript preparation.

\section{References}

Aparicio, E. H. (2009). Study biosaludables circuits for seniors in Spain: A study of geriatric parks for erderly people in Spain. Int. J. Med. Sci. Phys. Act. Sport, 9(33), 25-38.

Bedimo-Rung, A. L., Mowen, A. J., \& Cohen, D. A. (2005). The significance of parks to physical activity and public health: A conceptual model. Am. J. Prev. Med., 28, 159-168. https://doi.org/10.1016/j.amepre.2004.10.024

Besenyi, G. M., Kaczynski, A. T., Wilhelm Stanis, S. A., \& Vaughan, K. B. (2013). Demographic variations in observed energy expenditure across park activity areas. Prev. Med., 56(1), 79-81. https://doi.org/10.1016/j.ypmed.2012.10.011

Bettencourt, L., \& Neves, R. (2012). Seniors' playground and physical activity: Perceptions and practices. J. Aging Phys. Act., 20, S276.

Chow, H. W. (2013). Outdoor fitness equipment in parks: A qualitative study from older adults' perceptions. BMC Public Health, 13, 1216. https://doi.org/10.1186/1471-2458$13-1216$

Chow, H. W., Mowen, A., \& Wu, G. L. (2017). Who Is Using Outdoor Fitness Equipment and How? The Case of Xihu Park. Int. J. Environ. Res. Public Health, 14(4), 448. https://doi.org/10.3390/ijerph14040448

Cohen, D. A., Han, B., Isacoff, J., Shulaker, B., Williamson, S., Marsh, T., ... Bhatia, R. (2015). Impact of park renovations on park use and park-based physical activity. J. Phys. Act. Health, 12(2), 289-296. https://doi.org/10.1123/jpah.2013-0165

Cohen, D. A., Marsh, T., Williamson, S., Derose, K., Martinez, H., Setodji, C., \& McKenzie, T. L. (2010). Parks and physical activity: Why are some parks used more than others? Prev. 
Med., 50, S9-12. https://doi.org/10.1016/j.ypmed.2009.08.020

Cohen, D. A., Marsh, T., Williamson, S., Golinelli, D., \& McKenzie, T. L. (2012). Impact and cost-effectiveness of family fitness zones: A natural experiment in urban public parks. Health Place, 18(1), 39-45. https://doi.org/10.1016/j.healthplace.2011.09.008

Cohen, D. A., Marsh, T., Williamson, S., Han, B., Derose, K. P., Golinelli, D., \& McKenzie, T. L. (2014). The potential for pocket parks to increase physical activity. Am. J. Health Promot, 28, S19-S26. https://doi.org/10.4278/ajhp.130430-QUAN-213

Cranney, L., Phongsavan, P., Kariuki, M., Stride, V., Scott, A., Hua, M., \& Bauman, A. (2016). Impact of an outdoor gym on park users' physical activity: A natural experiment. Health Place, 37, 26-34. https://doi.org/10.1016/j.healthplace.2015.11.002

Dogru, E., Kizilci, M. H., Balci, N. C., Korkmaz, N. C., \& Tekindal, M. A. (2015) Investigation of exercise awareness and habits of individuals using outdoor fitness equipment. J. Exerc. Ther. Rehabil., 2, 102-109.

Duncan, M. J., Spence, J. C., \& Mummery, W. K. (2005). Perceived environment and physical activity: A meta-analysis of selected environmental characteristics. Int J Behav Nutr Phys Act, 2, 11. https://doi.org/10.1186/1479-5868-2-11

Evenson, K. R., Birnbaum, A. S., Bedimo-Rung, A. L., Sallis, J. F., Voorhees, C. C., Ring, K., \& Elder, J. P. (2006). Girls' perception of physical environmental factors and transportation: reliability and association with physical activity and active transport to school. The International Journal of Behavioral Nutrition and Physical Activity, 3, 28. https://doi.org/ $10.1186 / 1479-5868-3-28$

Gliner, J. A., Morgan, G. A., \& Leech, N. L. (2016). Research Methods in Applied Settings an Integrated Approach to Design and Analysis (3rd ed.). New York, USA. https://doi.org/10.4324/9781315723082

Godbey, G., \& Mowen, A. (2010). The Benefits of Physical Activity Provided by Park and Recreation Services: The Scientific Evidence. National Recreation and Park Association: Ashburn, VA, USA.

Haskell, W. L., Lee, I. M., Pate, R. R., Powell, K. E., Blair, S. N., Franklin, B. A., ... Bauman, A. (2007). Physical activity and public health: updated recommendation for adults from the American College of Sports Medicine and the American Heart Association. Med Sci Sports Exerc., 39(8), 1423-34. https://doi.org/10.1249/mss.0b013e3180616b27

Humpel, N., Owen, N., \& Leslie, E. (2002). Environmental factors associated with adults' participation in physical activity: A review. Am. J. Prev. Med., 22(3), 188-199. https://doi.org/ 10.1016/s0749-3797(01)00426-3

Kaczynski, A. T., Stanis, S. A. W., Hastmann, T. J., \& Besenyi, G. M. (2011). Variations in observed park physical activity intensity level by gender, race, and age: Individual and joint effects. J. Phys. Act. Health, 8, S151-S160. https://doi.org/10.1123/jpah.8.s2.s151 


\section{Macrothink}

Journal of Educational Issues ISSN 2377-2263

2021, Vol. 7, No. 3, Special Issue

Kershaw, C., Lim, J., McIntosh, J., Cornwall, J., \& Marques, B. (2017). Developing Resilience, Independence and Well-Being in Older Adults through Interactive Outdoor Spaces. Victoria University of Wellington: Wellington, New Zealand.

Laforest, S., Robitaille, Y., Lesage, D., \& Dorval, D. (2001). Surface characteristics, equipment height, and the occurrence and severity of playground injuries. Inj Prev., 7(1), 35-40. https://doi.org/10.1136/ip.7.1.35

Larson, L. R., Jennings, V., \& Cloutier, S. A. (2016). Public parks and wellbeing in urban areas of the United States. PLoS ONE, 11(4), e0153211. https://doi.org/10.1371/journal.pone. 0153211

Lin, B. B., Fuller, R. A., Bush, R., Gaston, K. J., \& Shanahan, D. F. (2014). Opportunity or orientation? Who uses urban parks and Why. PLOS ONE, 9(1), e87422. https://doi.org/ 10.1371/journal.pone.0087422

Madren, C. (2013). Hit the outdoor gym. Parks Recreat., 48, 40-46.

McKenzie, T. L., Cohen, D. A., Sehgal, A., Williamson, S., \& Golinelli, D. (2006). System for observing play and recreation in communities (SOPARC): Reliability and feasibility measures. J. Phys. Act. Health, 3, S208-S222. https://doi.org/10.1123/jpah.3.s1.s208

McLeroy, K. R., Bibeau, D., Steckler, A., \& Glanz, K. (1988). An ecological perspective on health promotion programs. Health Educ. Behav., 15, 351-377. https://doi.org/10.1177/ 109019818801500401

Mora, R. (2012). Moving Bodies: Open Gyms and Physical Activity in Santiago. Journal of Urban Design, 17(4), 485-497. https://doi.org/10.1080/13574809.2012.706367i

Mott, A., Evans, R., Rolfe, K., Potter, D., Kemp, K. W., \& Sibert, J. R. (1994). Patterns of injuries to children on public playgrounds. Arch. Dis. Child., 71(4), 328-330. https://doi.org/ 10.1136/adc.71.4.328

Nelson, M. E., Rejeski, W. J., Blair, S. N., Duncan, P. W., Judge, J. O., King, A. C., ... Castaneda-Sceppa, C. (2007). Physical activity and public health in older adults: recommendation from the American College of Sports Medicine and the American Heart Association. Med Sci Sports Exerc., 39(8), 1435-45. https://doi.org/10.1249/mss.0b013e $3180616 a a 2$

Onge, S. J. M., \& Krueger, P. M. (2011). Education and racial-ethnic differences in types of exercise in the United States. J Health Soc Behav, 52(2), 197-211. https://doi.org/ $10.1177 / 0022146510394862$

Rohde, C. L. E., \& Kendle, A. D. (1997). Nature for People. In A. D. Kendle, \& S. Forbes (Eds.), Urban Nature Conservation-Landscape Management in the Urban Countryside (pp. 319-335). E and FN Spon, London.

Sallis, J. F., Bauman, A., \& Pratt, M. (1998). Environmental and policy interventions to promote physical activity. Am J Prev Med., 15(4), 379-97. https://doi.org/10.1016/s0749- 
3797(98)00076-2

Sibson, R., Scherrer, P., \& Ryan, M. M. (2018). I think it adds value, but I don't use it: Use, perceptions and attitudes of outdoor exercise equipment in an urban public park. Annals of Leisure Research, 21(1), 58-73. https://doi.org/10.1080/11745398.2017.1290535

Xie, J. (2012). An analysis of the injury accidents of fitness route in non-central city, Sichuan Province. Sports Cult. Guide, 10, 82-84.

Yan, Y. (2016). Common problem analysis of outdoor fitness equipment. Technol. Wind, 19, 106.

\section{Copyright Disclaimer}

Copyright for this article is retained by the author(s), with first publication rights granted to the journal.

This is an open-access article distributed under the terms and conditions of the Creative Commons Attribution license (http://creativecommons.org/licenses/by/3.0/). 